\title{
Universal Adhesives- Different Curing Methods and Applications
}

\author{
Wanying Dong ${ }^{1, \mathrm{a}, \uparrow, *}$, Xiaojing $\mathrm{Gu}^{2, \dagger}$, Jianghao $\mathrm{Han}^{3, \mathrm{~b}, \uparrow, *}$, , Liwen You ${ }^{4, \dagger}$ \\ ${ }^{1}$ Chemical Engineering, Guangdong Technion-Israel Institute of Technology, 515063, Shantou, China \\ ${ }^{2}$ Polymer material science and engineering, Beijing University of Chemical Technology, 100029, Beijing, China \\ ${ }^{3}$ College of Chemistry, Nankai University, 300071, Tianjin, China \\ ${ }^{4}$ School of Materials Science and Engineering, East China University of Science and Technology, 201424, Shanghai, China \\ ${ }^{\dagger}$ These authors contributed equally to this work.
}

\begin{abstract}
The adhesive is a kind of material with good adhesion properties which could join objects by adhesion of surfaces via forces of adhesion and cohesion. There are many kinds of adhesives. This paper mainly classifies them by curing method, from UV curing and heat curing to moisture curing and microwave curing, and describes the corresponding mechanisms. Furthermore, it covers the advantages and disadvantages, application ranges, and related chemical compounds of different curing ways for adhesives. This can broaden our understandings of different ways of adhesive curing. Besides, it could also help us choose the most appropriate curing method according to different requirements in daily life.
\end{abstract}

\section{Introduction}

Adhesives have played a leading role in structural materials due to their excellent properties, including high bonding reliability and simple operation process [1]. They are commonly used in anti-corrosive materials, biomedical materials. For instance, super-glue fuming is used in police crime investigations to detect potential fingerprints [2]. Moreover, they also have a place in the military field. Adhesives can be used in tank maintenance as they are faster and better than welding. However, because of its brittleness, how to cure the different adhesives has been a hot spot in this field nowadays [3].

The curing process is the most vital part of the adhesive characteristics, which stands as one of the most significant ways to judge the usage of one kind of adhesive. It depends on the circumstance where we want to apply the adhesive, how we store it, and whether we can get rid of it after using it. Different conditions in need of adhesive spawn diversified requirements of them. Therefore, clarifying and analysing the curing method is an important academic topic in this area, both in the past and the future. The theory developed for the systematization of curing has gone through for several years, trying to improve our understanding of the curing process. By focusing on the factors that lead to the transformation, the curing method can be mainly divided into light-initiated curing, thermosetting curing, moisture curing, etc. Mostly, light-initiating curing is activated by high-energy ultraviolet ray photons, while heating plays the role of activator in the thermosetting curing process. Moisture curing is commonly used in mild conditions, as the energy to initiate the reaction is supplied by the adhesive molecule itself instead of provided by extraneous factors.

Adhesive curing is a process in which the properties of adhesives are obtained and improved through chemical reactions (polymerization, crosslinking, etc.) [4]. According to the curing way, the types of curing agents can be divided into reaction adhesive, solvent sensitive adhesive, UV curing adhesives, and anaerobic adhesive. Firstly, reaction adhesive is mainly classified into thermosetting resin type, thermoplastic resin type, synthetic rubber type, and mixed type. It can react chemically under certain conditions. A solidified adhesive layer is further polymerized or cross-linked into a linear or body shape structure. Secondly, solventsensitive adhesives are adhesives containing volatile organic solvents. This adhesive dissolves the resin component in the solvent to give the adhesive fluidity and then becomes a kind of adhesive absorbed by the sticky place through the solvent volatilization. For example, sodium silicate, starch, vinyl resin adhesives, rubber adhesives [5]. Thirdly, UV curing adhesive is a one-component, solvent-free, UV, and visible curable adhesive and sealant that can be cured with a wide range of light sources, such as point, pulse, and high-density lamps. Finally, it comes to the anaerobic adhesive. It will not solidify when it is in contact with oxygen or air. Once it is isolated from air, coupled with the catalytic effect of the metal surface, it can quickly polymerize and solidify at room temperature, forming a firm bonding and good sealing. It can be stored for many years by the effect of oxygen. A liquid adhesive that remains liquid in the presence of air at room temperature and rapidly solidifies in isolation.

${ }^{*}$ Corresponding author. Email: ${ }^{\mathrm{a}}$ Wanying.dong@gtiit.edu.cn, ${ }^{\mathrm{b}} 1812259 @$ mail.nankai.edu.cn 
This review summarizes the different curing methods of adhesives so far, including four different ways, UV curing, heat curing, moisture curing, and microwave curing. This review discusses the mechanisms, application ranges, advantages, and disadvantages of different curing methods. This summary can deepen the understandings of different ways of adhesive curing. Besides, we can choose the most appropriate curing method according to different requirements in industrial production and daily life.

\section{Curing methods}

\subsection{UV curing}

Ultraviolet curing (UV curing) is a processing technology that quickly cures the liquid materials employing ultraviolet light [6]. Under the irradiation of ultraviolet light, the polymerization of liquid adhesive material can quickly happen as the reactive substances are excited by UV light. Therefore, the curing of adhesive materials is very rapid within a short time. UV curing adhesives are widely used, such as epoxy acrylate adhesives, polyurethane acrylate adhesives, and polyester acrylate adhesives [7]. Compared with other traditional adhesives, UV curing adhesives have the advantages of convenient operation and use, fast curing speed, high production efficiency, no pollution, and energy-effective. They have been successfully applied in many industrial assembly fields, especially those that require a rapid assembly industry.

Generally, for a UV curing adhesive system, different components are included, such as photo-initiators, prepolymers, reactive diluents, and other additives. The function of the photo-initiator is to absorb UV and initiate polymerization. The pre-polymer is the main component of the adhesives [8]. The molecular structure and molecular weight of the pre-polymer affect the final properties of the adhesive such as curing rate, flexibility, adhesion, hardness, and so on. Furthermore, the reactive diluent is used to adjust the system viscosity, and it also participates in the curing process. Other ingredients, including dyes, stabilizers, surfactants, etc., can be added in appropriate amounts according to the different applications of the adhesives.

The photo-initiator in the glue system is firstly excited by ultraviolet light to generate free radical active species. Then the polymerization process proceeds with the unsaturated pre-polymers and monomers through grafting or crosslinking processes to obtain a bodyshaped network structured polymer. The active monomer acts as a bridge, which links the pre-polymers with different molecular weights together to form long molecular chains. For example, diethylene glycol diacrylate is triggered by ultraviolet light to produce a polymer with a network structure (Fig. 1).

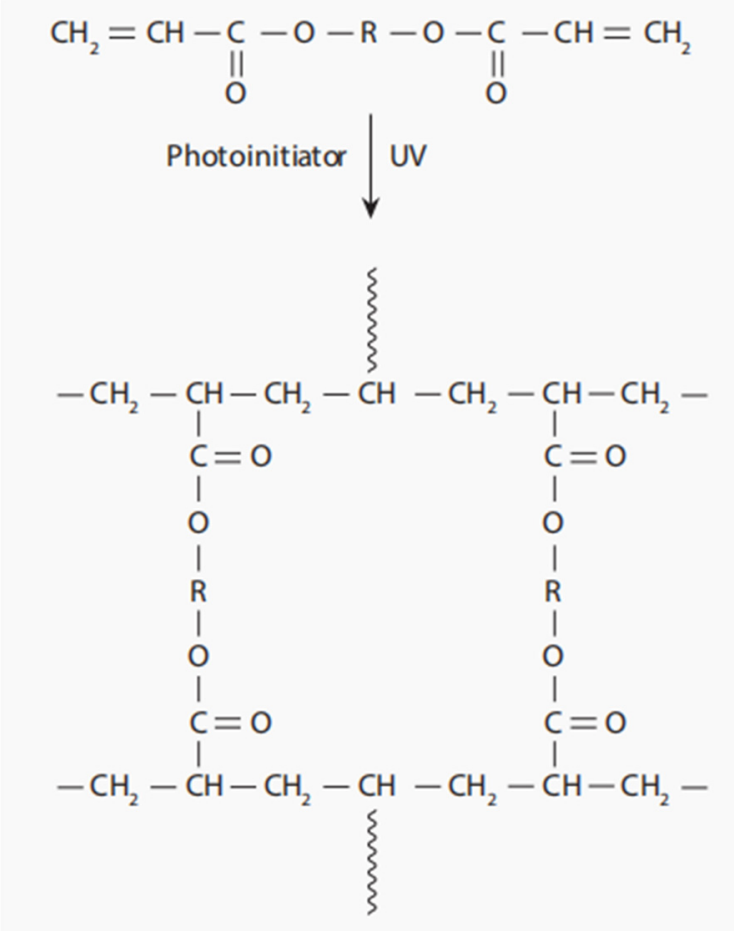

Fig. 1. Photocrosslinking of a diacrylate (simplified): formation of a network [8].

In general, UV curing adhesives have some advantages and disadvantages. Firstly, the process of UV curing can be carried out at room temperature, which indicates that UV curing is an energy-effective and costeffective process, and the required equipment for the curing process is relatively simple. Furthermore, there is no organic solvent in the system in UV curing adhesives, which is an environmental-friendly process. In contrast, thermoplastic adhesives are processed through endothermic physical changes (such as solvent evaporation and melting). Moreover, some low-volatility monomers are added to the adhesive system to adjust its viscosity, and these monomers can fully participate in the curing. These monomers act as a bridge to connect the oligomers and finally produce a cross-linked substance. As a result, the solid content can be regarded as $100 \%$ [9]. Finally, UV-cured adhesives can cure in a very short time. For example, superfast curing (in less than $0.5 \mathrm{~s}$ ) of acrylate adhesives for plastics has been achieved by a proper choice of pre-polymers, photoinitiators, and photo-sensitizers, which have been successfully used in blister packaging, as well as for bonding glass to plastics [6]. The reduced time of cure implies much higher productivity, which can increase profits and reduce costs. However, since UV light cannot pass through opaque materials, UV curing technology is not suitable for curing opaque systems. Furthermore, the light-curing rate is fast, the internal stress of the formed cured product is relatively large, and the strength and adhesion of the material will also be affected. Finally, the addition of monomers will also make the adhesive system have a greater shrinkage after curing. 


\subsection{Heating Curing}

Heat curing adhesive is a kind of heat curing crosslinked polymers or heats curing plastics. Most heating curing adhesives are supplied in a two-component system, usually made up of a resin and a hardener. They are usually existent as liquid form or gel form, and the curing process will initiate when they are mixed. The reaction time usually takes in a range between 10 minutes to a few hours. Moreover, there are also some adhesives are supplied in single-component systems or one-component systems. In this system, the heat curing pastes must be cold-stored to delay the curing itself for certain applications, such as phenolic resin. Onecomponent systems can offer advantages in some specific reaction processes. It is unnecessary to add any other additional substances into the system because all of the required components, say the main agent, curing agent and crosslinking agent, have been mixed within one component.

Epoxies are a class of the most common heat curing adhesives, and several different advantages are offered in distinct applications. For example, when used to bond the wood, better resistance to moisture and a higher ability in gap-filling are provided. And when it is employed to bond the metals and glass, epoxy resins are also unusual as they could create a strong bond. However, unmodified epoxy is a kind of hard and brittle solid, which is easily fractured from time to time. Consequently, it is vital to improving the toughness, flexibility, and fatigue resistance of adhesives. Ordinarily, different modifiers are utilized in most cases. For example, the addition of benzoic acid as a modifier could enhance the deoxidizing curing ability and compatibilization effect.

Additionally, some other polymers can be used as heat curing adhesives, such as polyester resin, which could be used as a lower-cost substitution to epoxy resins. However, the ability of it in gap filling and bond strength is not as outstanding as epoxy resins. Phenolic resins, one of the earliest forms of plastics, could also act as heat curing adhesive, typically used for paper and wood.

Different chemical reactions usually happen for the heat curing adhesives during the process of curing, which results in higher strength for obtained products. There are many other advantages as well. First of all, the gap filling ability is excellent. Moreover, the resistance to moisture, heat and other changes from the outside environment is also good. However, the impact strength and peel strength are sometimes low. In addition, the initial cohesiveness is not high. And the ability of water resistance is also a big problem that adhesives face nowadays. Hence, it is essential to add some modifications necessary in the process of curing to overcome these problems. For instance, Dai and coauthors have introduced a proper amount of dopamine and metal ion to improve the water resistance of the epoxy adhesives and endow them with excellent adhesion properties [10].

\subsection{Moisture curing adhesive}

In most common circumstances, the adhesive should be used in the open air, and the process of curing has to take place in a mild condition. An ideal situation is that the adhesive keeps liquid form in the bottle and turns hard as soon as being exposed to the air. $\mathrm{H}_{2} \mathrm{O}$ molecule has a strong potential of nucleophilic attacking. Therefore, the major strategy for moisture curing is to provide a structure that can quickly react with water.

One of the most effective methods is introducing an isocyanate group, which can immediately react with the moisture from the atmosphere [11]. Isocyanate groups can be attacked by nucleophilic reagents, including those molecules with -OH or - $\mathrm{NH}_{2}$ groups (Fig.2). And then, a decarboxylation process happens for carboxylic acid, and an anime is formed. It can then react with another isocyanate group. Eventually, the small molecules are linked together by an acylamide bond.

Like the alkoxy groups in alkoxysilanes, other kinds of groups are also able to realize such a similar process [12]. Firstly, silanol and alcohol are generated from the reaction. After that, the silanol group condenses together or catches other alkoxy groups to form the siloxane group, eliminating water or alcohol molecules (Fig.3). In this process, the $\mathrm{H}_{2} \mathrm{O}$ can reach deeper under the surface to react with mole alkoxy groups.

A type of glue that is widely used in our daily life is called ethyl cyanoacrylate. In this molecule, two electron-withdrawing groups are linked to the same carbon atom in the monomer, creating a high tendency to be attacked nucleophilic reagents. Therefore, a very little amount of $\mathrm{OH}$ - from the moisture in the air can initiate an anionic polymerization, quickly transferring the system into a kind of mass that is hard and dry. The curing process is just the process of polymerization.

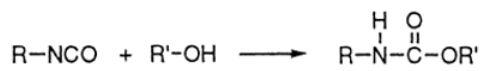

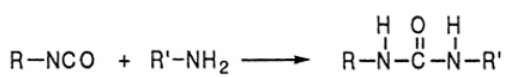

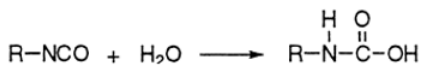

$$
\begin{aligned}
& \stackrel{\stackrel{\mathrm{H}}{\mathrm{O}} \mathrm{O}}{\mathrm{R}-\mathrm{N}-\mathrm{C}-\mathrm{OH}} \longrightarrow \mathrm{R}-\mathrm{NH}_{2}+\mathrm{CO}_{2}
\end{aligned}
$$

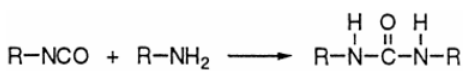

Fig.2. The whole process of the curing of compounds led by isocyanate groups. (3) take place as soon as facing the moisture, generating the substrate of (4). The anime that just formed can react with an unreacted NCO group (5), linking each other together $(\mathrm{S}[11]$.

$$
\begin{aligned}
& \equiv \mathrm{Si}-\mathrm{OR}+\mathrm{H}_{2} \mathrm{O} \rightarrow \equiv \mathrm{Si}-\mathrm{OH}+\mathrm{ROH} \\
& \equiv \mathrm{Si}-\mathrm{OH}+\equiv \mathrm{Si}-\mathrm{OH} \rightarrow \equiv \mathrm{Si}-\mathrm{O}-\mathrm{Si} \equiv+\mathrm{H}_{2} \mathrm{O} \\
& \equiv \mathrm{Si}-\mathrm{OR}+\equiv \mathrm{Si}-\mathrm{OH} \rightarrow \equiv \mathrm{Si}-\mathrm{O}-\mathrm{Si} \equiv+\mathrm{ROH}
\end{aligned}
$$

Fig.3. The mechanism of curing by alkoxysilane is shown in the graph. After reacting with the $\mathrm{H} 2 \mathrm{O}$ molecule, the group transfer into a silanol group, which is ready to build a Si-O-Si bond to construct the polymer [12]. 
The most vital characteristic of moisture curing is effectiveness. The conversion of adhesive can take place immediately when it is exposed to the moisture air. Significant advantage as it is, the promptness of moisture curing also acts as the greatest limitation to the usage of this kind of adhesives because the efficacy of it will lose as soon as being exposed to the air. Therefore, it is crucial to avoid any possibility of touching the moisture during the manufacturing, transporting, and storing process.

\subsection{Microwave curing}

Microwave curing is also widely utilized for the curing of adhesive, such as E44/DDM, UPR, and so on. Generally, the microwave frequency is about $0.3 \sim 300$ $\mathrm{GHz}$, and it will produce heating, melting even chemical reaction, and other physical phenomena with the assistance of microwave. Therefore, microwave curing is more efficient than the conventional methods. It can make chemical reactions more than 3 times or even dozens of times faster at the same or even lower temperature. Due to such high efficiency, microwave curing can also be used for chemical synthesis [13]. Furthermore, microwave curing can easily convert the liquid resin into a solid state, which could benefit the curing of adhesive.

K.Johnston and co-authors have carefully investigated the microwave curing mechanism of epoxy. As shown in Fig 4, firstly, the epoxy reacts with amines through a ring-opening mechanism. Then during the curing process, an epoxy group reacts with a primary amine $\left(\mathrm{RNH}_{2}\right)$ to produce a hydroxyl group and a secondary amine (RNH). In the second step, the formed secondary amine reacts with another epoxy group to produce a hydroxyl group and a tertiary amine. Opening of the epoxy ring by an amine to form $\mathrm{OH}$ and $\mathrm{CN}$ groups [14]. It seems that the chemical mechanism of microwave curing is essentially similar to that of heat curing. However, the rate of microwave curing is much faster. Thus, microwave curing has a more complex effect on the curing process of adhesive, which is obviously different from the effect of heating curing just by increasing the reaction temperature [15]. Microwave curing can reduce the curing time and manufacturing costs. Moreover, the consumed energy of microwave curing is obviously reduced.
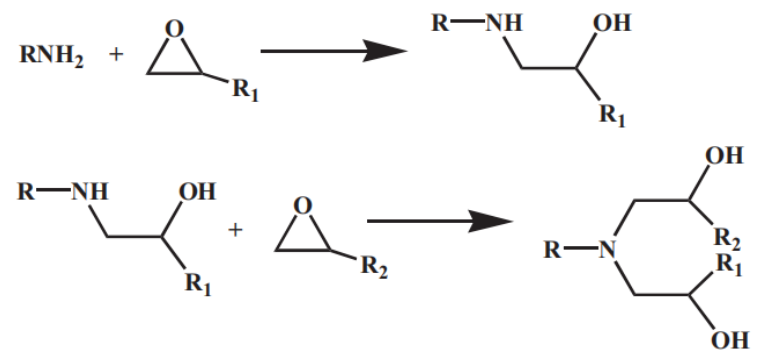

Fig.4. The general mechanism for curing of an epoxy resin with an amine curing agent [13]

\section{Conclusion}

This article has reviewed the research progress of adhesives through four curing methods: UV curing adhesives, heating curing adhesives, moisture curing adhesives, and microwave curing adhesives. These four curing methods have their own advantages and disadvantages. Moisture-curing adhesives are very easy to use. Compared with other curing methods, they do not require special equipment and can be cured in the air. However, moisture-curing adhesives are easily affected by humid environments and water, and they have poor resistance to polar solvents. In contrast, the curing process of thermal curing and UV curing adhesives requires specific equipment. Thermal curing adhesives have higher strength and excellent bonding performance. However, the curing speed is slow, and the curing process usually lasts from ten minutes to several hours. The curing speed of UV-curing adhesives is much faster than that of the thermal curing adhesives, and therefore it is more efficient, which is more conducive to industrial applications. Microwave curing is a new type of curing method that has been used in adhesives, and the curing speed is also extraordinarily fast. However, the high speed of the curing process also has a weakness. When the adhesive is cured, the volume shrinkage due to solvent volatilization or chemical reaction will generate internal stress, which will affect durability and bonding strength. As a result, though UV curing and microwave curing have higher efficiency, they will also cause greater internal stress in the material, thereby affecting the performance of adhesives. Most industrial applications have a great demand for adhesives. In future research, how to improve the bonding efficiency, bonding performance, and cost reduction of adhesives will be very important topics.

\section{References}

[1] S. Rimdusit, H. Ishida, Polymer 2000, 41(22), 79417949.

[2] R.C. Benson, D. Farrar, J.A. Miragliotta, Johns Hopkins APL Technical Digest (Applied Physics Laboratory) 2008, 28(1), 58-71.

[3] I. Fotiou, A. Baltopoulos, A. Vavouliotis, V. Kostopoulos, Journal of Applied Polymer Science, 2013, 129(5), 2754-2764.

[4] Eun Seon Kim, Jae Heung Lee, Dong Hack Suh, Woo Jin Choi, Macromolecular Research 2021, 29, 129-139

[5] Nasrin Moini,Maryam Khaghanipour,Kourosh Kabiri,Ali Salimi,Mohammad J. ZohuriaanMehr,Arash Jahandideh, 5. ACS Sustainable Chemistry \& Engineering 2019, 7, 19, 1624716256.

[6] DH Wang, L Jiang. Theory and Application of Ultraviolet Curing Materials [M]. Beijing: Science Press, 2001.

[7] YT Xue. Study on Preparation and Properties of UV-Curable Adhesive [D]. Fuzhou University ,2011. 
[8] V Alessandra, T Giuseppe, B Roberta. Reviews of Adhesion and Adhesives,2017,5(2).

[9] M Bajpai,V Shukla,A Kumar. J. Progress in Organic Coatings,2002,44(4), 271-278.

[10] F Dai, F. Chen, T. Wang, S. Feng, C. Hu, X. Wang, Z. Zheng Journal of materials Science, 2016, 51, $4320-4327$

[11] Comyn and F Brady and R.A Dust and M Graham and A Haward. International Journal of Adhesion and Adhesives, 1998, 18(1), 51-60.

[12] Hai, N. , A. D. Skaja , and MD Soucek. J.Progress in Organic Coatings 2000,40(1), 175-184.
[13] Johnston K, Pavuluri S K, Leonard M T, Thermochimica Acta, 2015, 616(20), 100-109.

[14] S.K. Pavuluri, M.P.Y. Desmulliez, G. Goussetis, et al. Post cure behaviour of encapsulants for QFNpackages processed by an open-ended single mode resonant microwaveapplicator, $2011 \mathrm{IEEE}^{13}$ th Electronics Packaging Technology Conference(EPTC), 2011, 573-578.

[15] R.C.Benson, D. Farrar, J.A. Miragliotta. Polymer adhesives and encapulants formicroelectronic application, Johns Hopkins Apl Tech. Dig. 2008, 28, 58-71. 